\title{
Shaping protein distributions in stochastic self-regulated gene expression networks
}

\author{
Manuel Pájaro, ${ }^{1, *}$ Antonio A. Alonso, ${ }^{1, \dagger}$ and Carlos Vázquez ${ }^{2} \ddagger$ \\ ${ }^{1}$ Process Engineering Group, IIM-CSIC. Spanish Council for Scientific Research. \\ Eduardo Cabello 6, 36208 Vigo, Spain \\ ${ }^{2}$ Departament of Mathematics, Faculty of Informatics. \\ Campus de Elviña, s/n 15071 - A Coruña, Spain
}

(Dated: August 31, 2015)

\begin{abstract}
In this work, we study connections between dynamic behavior and network parameters, for selfregulatory networks. In that aim, a method to compute the regions in the space of parameters that sustain bimodal/binary protein distributions has been developed. Such regions are indicative of stochastic dynamics manifested either as transitions between absence and presence of protein or between two positive protein levels. The method is based on the continuous approximation of the Chemical Master Equation, unlike other approaches that make use of a deterministic description, which as it will be shown can be misleading.

We find out that bimodal behavior is a ubiquitous phenomenon in cooperative gene expression networks under positive feedback. It appears for any range of transcription and translation rate constants whenever leakage remains below a critical threshold. Above such threshold, the region in the parameters space which sustains bimodality persists, although restricted to low transcription and high translation rate constants. Remarkably, such threshold is independent of the transcription/translation rates or the proportion of active/inactive promoter and depends only on the level of cooperativity.
\end{abstract}

The proposed method can be employed to identify bimodal/binary distributions leading to stochastic dynamics with specific switching properties, by searching inside the parameters regions that sustain such behavior.

Keywords: self-regulated gene expression networks, positive feedback, cooperativity, stochastic switchers, critical leakage, bimodal/binary distributions

\footnotetext{
*mpajaro@iim.csic.es
} 


\footnotetext{
$\dagger$ Author to whom correspondence should be addressed. E-mail: antonio@iim.csic.es $\ddagger$ carlosv@udc.es
} 


\section{INTRODUCTION}

The underlying biochemical reactions responsible for transcription, translation and regulation in genetic networks usually involve reduced numbers of molecules. Under these conditions, chemical interactions are far away from the classical kinetic limit [1, 2], thus making gene expression a truly stochastic process.

Over the past decade, many studies reported evidences of the role of intrinsic noise in intracellular processes as the major cause of heterogeneous response in individual cells [3-6]. In [7], stochasticity in gene expression as a source of cell differentiation has been reviewed. This study emphasized the positive consequences derived from the adaptability to fluctuating environments, rather than the detrimental effects concerning cellular malfunction (and thus disease). Different works [8-11] provided experimental evidence of such behavior in bacteria and eukaryotic cells, both at the cell populations level and in single cells.

Linking experimental data with network structure and functionality requires a quantitative characterization of intrinsic and extrinsic noise sources [12-14]. In this setting, mathematical modelling of gene expression emerged and provided deterministic and stochastic descriptions. Deterministic models are mainly based on classical biochemical kinetics, such as in [15] or [16]. Stochastic approaches range from those based on the chemical master equation (CME) [2, 17-20], to the ones developed in terms of stochastic differential equations (e.g. [21, 22]). Over the last years, mathematical modelling has been combined with experiments to elucidate mechanisms and to produce models with predictive capabilities (e.g. $[20,23,24])$.

Conceptually, gene expression networks can be modelled as the assembly of simple transcription-translation structures linked by a diversity of regulatory mechanisms. Such fundamental structures, conceived as efficient abstractions of the central dogma [20], will carry different functions in the cell [16]. Regulatory functions produced by negative or positive feedback are among the most common.

Negative feedback has been observed to reduce noise, but also to induce sustained oscillations under time delays [7]. In living organisms, this fact confirms a typically predicted result in classical control systems theory. On the other hand, it has been shown that the positive feedback amplifies fluctuations and induces bimodal responses in the form of state switching transitions, as expected from the theory. 
In a biological context, this function offers the possibility of sampling different physiological states, thus increasing the chances of survival without the need for a genetic mutation. Evidence of such mechanism in bacteria has been reported in e.g. [25] or [26]. The first work demonstrates that bacterial populations synchronize phenotype switching to the frequency of environmental fluctuations. The second study (experimentally) proves that switching transitions between a noncompetent and a competent state can be tuned by redesigning the corresponding gene regulatory network.

The present study mainly focus on self-regulatory networks, namely those in which the expressed protein regulates its own production. More precisely, we examine in a stochastic context all possible dynamical regimes that can be sustained by this structure. For this purpose, a method that relates stochastic behaviour to network parameters is here developed. The method computes the regions in the parameters space that lead to bimodal/binary stationary distributions, in the understanding that such distributions correspond to transitional dynamics switching between the two most frequent states. Note that binary distributions imply transitions from absence to presence of protein, while bimodality reflects transitions between different protein levels. Both regimes oppose to graded distributions which allow just one state to be the most frequently visited.

Deterministic descriptions based on ODE (ordinary differential equation) systems have been previously employed to identify the regions in the parameters space exhibiting bistable responses for such class of networks (e.g. [27]). Such behaviour could be understood as the deterministic counterpart of transitional dynamics in stochastic systems (see for instance [28]). However, as we will discuss further in this work, one can find many instances in which a bimodal/binary stationary distribution associated with the stochastic system will correspond to a monostable deterministic counterpart.

Central to the proposed method is a partial integro-differential equation (PIDE) firstly proposed in [29] which approximates the behaviour of the network reasonably well and provides amenable solutions for analysis. As reported in the literature [29-31], the validity of such an approximation extends to networks where proteins are produced in episodic bursts rather than by constitutive expression. This scenario requires that the messenger RNA degrades faster than proteins, what is often the case for many prokaryotic and eukaryotic organisms $[30,32]$. This condition has been recently reported as the main cause of heterogeneity in the context of embryonic stem cells [11]. 
Concerning some recently addressed questions on bimodality [27, 31, 33], we found out that it is an ubiquitous phenomena in cooperative gene expression networks under positive feedback. In fact, it is present for any range of transcription and translation rate constants, provided that leakage remains below a critical threshold. Above such threshold, the region in the parameters space which sustains transitional dynamics persists, although withdrawn to low transcription and high translation rate constants. Remarkably, such threshold is independent of the transcription/translation rates or the proportion of active/inactive promoter and depends only on the level of cooperativity (i.e. number of binding sites in the promoter). The field of synthetic biology may benefit from the knowledge of the relationships between network parameters and dynamics. Understanding the stochastic dynamics may guide the development of methods for noise reduction or help to overcome the inherent uncertainty about the values of the parameters (see for instance the pioneering work in [34]). Together with the design of the appropriate RNAses or proteases to modulate network function $[11,35]$, those questions remain among the most critical obstacles found in practical applications, such as the construction of genetic biosensors (e.g. [5]).

\section{SYSTEM DESCRIPTION AND REPRESENTATION}

The network under study consists of a typical transcription-translation mechanism involving a single gene that expresses a given protein and regulates its own production. The representative biochemical steps, including protein and $m R N A$ degradation, are depicted in Figure 1.

As proposed in [29] and recently discussed in [31], we assume a basal transcription level (also known as transcriptional leakage) from the inactive promoter, occurring with a rate constant $k_{\varepsilon}$ lower than $k_{1}$.

In this study, we consider gene expression networks with $m R N A$ degradation rates being much faster than the corresponding protein degradation ones, so that $\gamma_{1} / \gamma_{2} \gg 1$. Such condition will result into protein evolving in episodic bursts, what seems to be more often the case both in prokaryotic and eukaryotic cell types [32]. Experimental evidence of such bursts has been reported in $[8,9,30,36]$, among others. A recent study shows that episodic bursts are particularly frequent along the human genome [32]. Such phenomena have been also recently reported in [11] as the main cause of heterogeneity in the context of embryonic 
stem cells.

\section{A. The self-regulation mechanism}

The activation/inactivation of genes is assumed to be determined by the binding of their own expressed protein molecules in what constitutes a feedback regulation loop. In this study, we will consider both negative and positive feedback scenarios. In the former, protein binding inhibits its own production whereas in the latter production is activated. Let $n$ be the number of proteins that bind to a given promoter, and assume that binding takes place by reversible bimolecular reaction steps so that:

$$
D N A_{i-1}+X \underset{k_{i}^{-}}{\stackrel{k_{i}^{+}}{\rightleftharpoons}} D N A_{i}
$$

where the subscript $i$ indicates the number of protein molecules already attached and ranges from $i=1, \cdots, n$. Constants $k_{i}^{+}$and $k_{i}^{-}$are the rates per unit time associated to the $i$

forward and backwards reaction channel, respectively. If we define $K_{i}=\frac{k_{i}^{-}}{k_{i}^{+}}$for $i=1, \cdots, n$ and assume that $K_{i}$ for $i=1, \cdots, n-1$ to be large enough, then the presence of the intermediate species $(i=1, \cdots, n-1)$ is negligible, what results into $D N A_{0}$ and $D N A_{n}$ to

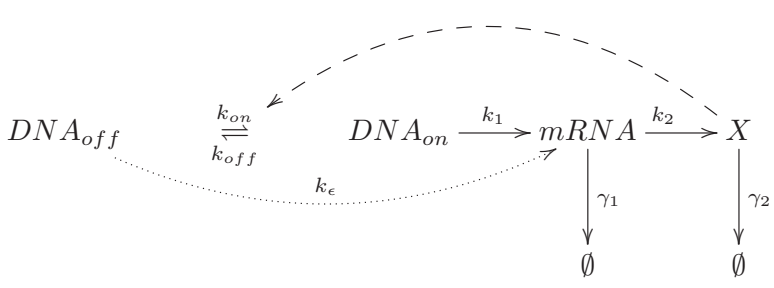

FIG. 1. Schematic representation of the transcription-translation mechanism under study. The promoter associated to the gene of interest is assumed to switch between active $\left(D N A_{\text {on }}\right)$ and inactive $\left(D N A_{\text {off }}\right)$ states, with rate constants $k_{\text {on }}$ and $k_{\text {off }}$ per unit time, respectively. In this study, the transition is assumed to be controlled by a feedback mechanism induced by the binding/unbinding of a given number of $X$-protein molecules, what makes the network self-regulated. Transcription of messenger RNA ( $m R N A$ ) from the active $D N A$ form, and translation into protein $X$ are assumed to occur at rates (per unit time) $k_{1}$ and $k_{2}$, respectively. $k_{\varepsilon}$ is the rate constant associated to transcriptional leakage. Both $m R N A$ and $X$-protein degradation are assumed to occur by first order processes with rate constants $\gamma_{1}$ and $\gamma_{2}$, respectively. 
be the most probable states [16]. Depending on whether the feedback is negative or positive, $D N A_{\text {on }}$ will correspond with $D N A_{0}$ or $D N A_{n}$, respectively. Nonetheless, the probability of each state is related to the amount of protein that activates or inactivates the promoter. Such probability is given by a function $\rho: \mathbb{R}_{+} \rightarrow[0,1]$ of the Hill type, that can be defined in terms of the fraction of $D N A_{\text {off }}$ as:

$$
\rho(x)=\frac{x^{H}}{x^{H}+K^{H}}
$$

where $x$ represents the amount of expressed protein (defined either as numbers of protein molecules or protein concentration). The integer number $H$ is proportional to the number of protein molecules bound to the promoter. The positive or negative sign of $H$ depends on whether protein inhibits or promotes transcription, what corresponds to negative and positive feedback, respectively ( $H=0$ would indicate the network is open loop).The constant $K$ in expression (2) is related to the forward and backward reaction step constants by $K=\sqrt[|H|]{\prod_{i=1}^{n} K_{i}}$. Under feedback, the transcription rate $R_{\mathrm{T}}$ (which represents the rate of production of $m R N A$ ) is essentially proportional to the fraction of active promoter, so that $R_{\mathrm{T}} \equiv k_{1} \bar{c}(x)$, with:

$$
\bar{c}(x)=[1-\rho(x)]+\rho(x) \varepsilon .
$$

The above expression includes a small parameter $\varepsilon=k_{\varepsilon} / k_{1}$ that accounts for the contribution of basal transcription (leakage) to the total rate. In line with [31], we employ this expression instead of the one suggested in [29], which reads $c(x)=1-\rho(x)+\varepsilon$. Of course $c(x)$ will result in a good approximation of $\bar{c}(x)$ when the fraction of active $D N A_{\text {on }}$ becomes small.

\section{B. Modelling the dynamics}

As discussed in e.g. [17], gene expression is inherently stochastic, so it calls for stochastic modelling approaches. In this work, we make use of the framework offered by the Chemical Master Equation (CME) to describe the dynamics of the species that constitute the network depicted in Figure 1.

In constructing the CME, the promoter is assumed to be in $n$ possible configurations that coincide with $D N A_{\text {on }}, D N A_{\text {off }}$ and the intermediate binding forms $D N A_{i}$ previously described. A 3-dimensional vector $\mathbf{n}$ specifies the dynamical state of the system with components representing the promoter configuration, and the number of molecules for $m R N A$ 
and $X$-protein.

In this work, the CME of the network has been numerically solved with a finite state projection algorithm [19], adapted to the accuracy requirements for each of the considered scenarios. Specific realizations of the network dynamics were produced with the Gillespie Stochastic Simulation Algorithm (SSA) [37]. This algorithm has also been employed to assess the accuracy of the CME approximations, by computing a statistically representative number of system's realizations to reconstruct the corresponding probability distributions.

In order to make possible the analysis of the stochastic dynamics, we will employ the continuous approximation proposed in [29] for the corresponding CME that describes the temporal evolution of protein distribution, $p: \mathbb{R}_{+} \times \mathbb{R}_{+} \rightarrow \mathbb{R}_{+} \backslash\{0\}$, in gene expression networks. The following PIDE was proved to be an appropriate approximation under the assumption that proteins in the network are produced by bursts:

$$
\frac{\partial p(\tau, x)}{\partial \tau}=\frac{\partial}{\partial x}(x p(\tau, x))+a \int_{0}^{x} w\left(x-x^{\prime}\right) \bar{c}\left(x^{\prime}\right) p\left(\tau, x^{\prime}\right) \mathrm{d} x^{\prime}
$$

where $\tau=\gamma_{2} t$ represents a dimensionless time associated to the time scale of protein degradation. The first term in the right-hand side of the equation accounts for protein degradation whereas the integral describes protein production by bursts. The size of the bursts is assumed to follow an exponential distribution [38]. More precisely, the conditional probability for protein level to jump from a state $x^{\prime}$ to $x$ after a burst is given by the following expression [29]:

$$
w\left(x-x^{\prime}\right)=(1 / b) \exp \left(\left(x^{\prime}-x\right) / b\right)-\delta\left(x-x^{\prime}\right)
$$

Parameters $a=k_{1} / \gamma_{2}$, in Eqn (4), and $b=k_{2} / \gamma_{1}$, in Eqn (5), are dimensionless rate constants associated to transcription and translation, respectively. In order to model the feedback mechanism, function $\bar{c}$, as defined in Eqn (3), is included within the integral.

There is a considerable amount of experimental evidence confirming that protein synthesis occurs in bursts (e.g. $[9,11,30]$ ), provided that the mRNA degradation rate is much higher than the one corresponding to proteins, thus supporting the validity of expression (4).

As an example, Figure 2 compares numerical solutions of Eqn (4), both for transient and stationary regimes, with the corresponding distributions obtained by using the CME and also with the stochastic simulations based on the SSA Gillespie algorithm. In all cases, the PIDE approximation shows an excellent agreement with the alternative descriptions. 


\section{BIMODAL/BINARY DISTRIBUTIONS IN STOCHASTIC GENE EXPRES-} SION

In this section, we study conditions under which the stochastic behavior of a transcriptiontranslation network can be described by a bimodal/binary distribution, corresponding to a transitional dynamics switchings between two most frequent protein levels. In that purpose, we first characterize the stationary distributions as a function of the network parameters. Secondly, we find the region in the parameters space of the network that leads to bimodal/binary distributions.

\section{A. Feasible stationary distributions}

The stationary solution for Eqn (4) (identified as the one satisfying the equation with $\left.\frac{\partial p}{\partial \tau}=0\right)$, is denoted by $P$ and takes the form:

$$
P(x)=C(\rho(x))^{\frac{a(1-\varepsilon)}{H}} x^{-(1-a \varepsilon)} e^{\frac{-x}{b}},
$$

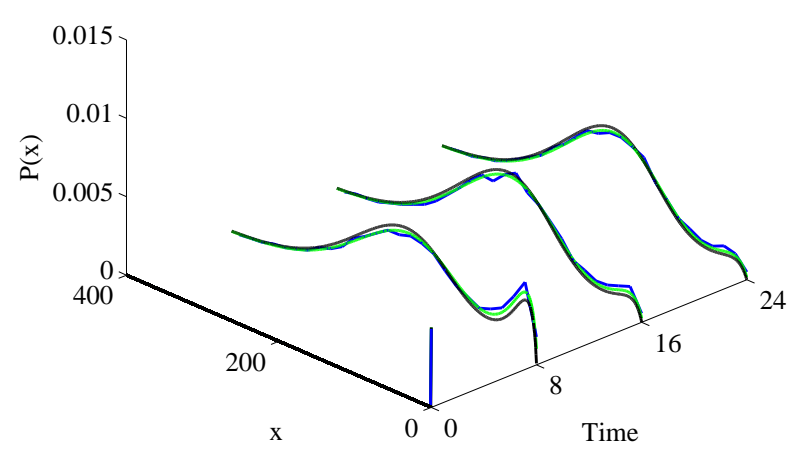

FIG. 2. Transient distributions for positive feedback $(H=-4)$ with leakage $\varepsilon=0.2$. In order to compare accuracy, distributions were computed from CME (green lines), Eqn (4) (black lines) and Gillespie SSA realizations (in the order of 10000) to reconstruct the distributions (blue lines). Parameters employed to simulate the self-regulation mechanism are presented in Table I. The initial condition is given by a delta function centered at $x=0$ to represent the absence of protein at $\tau=0$. The snapshot at the right of the plot corresponds to a stationary distribution. (Color Online). 


\begin{tabular}{ll} 
Parameter Value \\
\hline$\gamma_{1}$ & $0.01 s^{-1}$ \\
$\gamma_{2}$ & $0.0004 s^{-1}$ \\
$a$ & 10 \\
$b$ & 20 \\
$K$ & $70 n M$ \\
$\varepsilon$ & 0.2 \\
$K_{+}$ & $1 n M^{-1} s^{-1}$ \\
$k_{+}^{4}$ & $1 n M^{-1} s^{-1}$ \\
$k_{-}^{4}$ & $3 s^{-1}$ \\
$k_{+}^{3}$ & $5 n M^{-1} s^{-1}$ \\
$k_{-}^{3}$ & $15 s^{-1}$ \\
$k_{+}^{2}$ & $10 n M^{-1} s^{-1}$ \\
$k_{-}^{2}$ & $30 s^{-1}$ \\
$k_{+}^{1}$ & $\left(K_{+}\right)^{4} /\left(k_{+}^{2} k_{+}^{3} k_{+}^{4}\right)$ \\
$k_{-}^{1}$ & $\left(K_{-}\right)^{4} /\left(k_{-}^{2} k_{-}^{3} k_{-}^{4}\right)$ \\
$k_{\varepsilon}$ & $\varepsilon k_{1}$ \\
&
\end{tabular}

TABLE I. Reaction constants employed to simulate protein binding in reaction steps (1) for $H=$ -4 . The values for the constant $K$ and the leakage $\varepsilon$ have been taken from [29]. The value for $K^{+}$ has been taken from [16].

where $C$ is an integration constant that normalizes the corresponding pdf (probability distribution function) so that $\int_{0}^{\infty} P(x) \mathrm{d} x=1$. Details on the derivation of (6) are presented in Appendix A. Essentially, there are two types of stationary distributions that correspond with two transitional stochastic regimes:

- Bimodal distributions, with two maxima, which characterize transitions between two positive protein levels.

- Binary distributions, with a positive minimum and maximum, characterizing transitions between a positive and a zero level, denoting the absence of protein. 
(A)
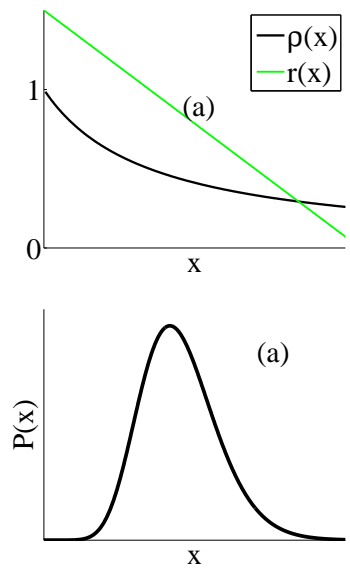

(B)

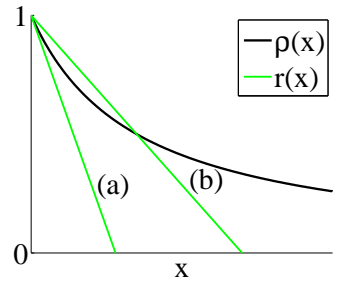

(a)
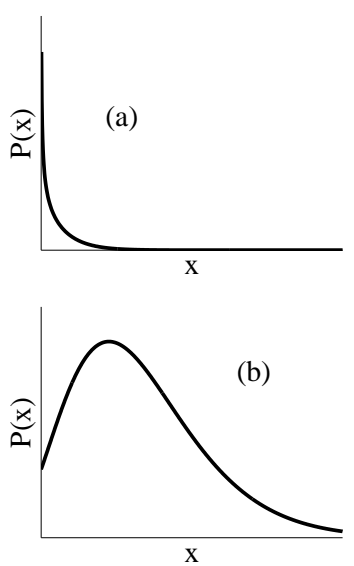

(C)

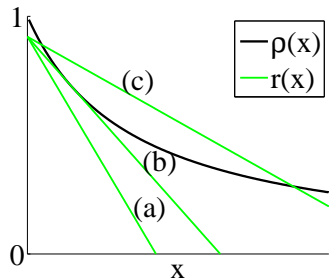

(a)

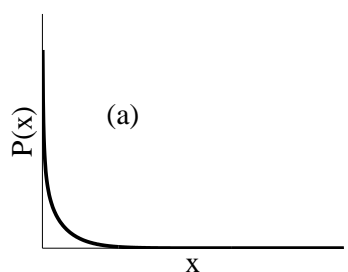

(b)

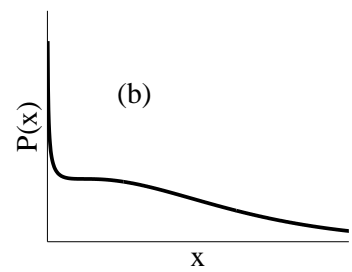

(c)

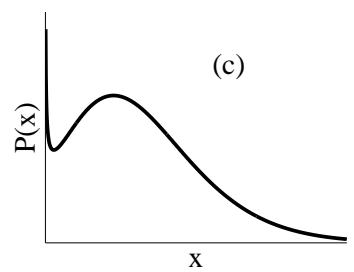

(D)

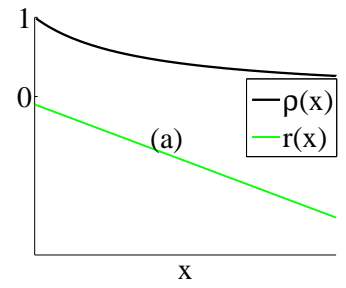

(a)

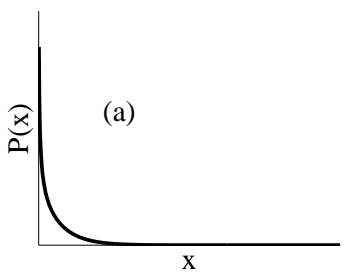

FIG. 3. The qualitative shape of all possible distributions for $H=-1$ depending on the intersections between $\rho(x)$ and $r(x)$ in Eqn (8), (black and green lines, respectively, on the first column plots). The different $r(x)$ are marked with a letter to identify which shape corresponds to each line. For $r(0) \geq 1$ there is at most one positive intersection that corresponds with graded responses (rows $\mathrm{A}$ and $\mathrm{B})$. For $0<r(0)<1$ there can be two positive intersections leading to binary distributions (row C). For $r(0) \leq 0$ no intersection occurs (row D). (Color Online).

In order to identify bimodal/binary distributions, we first study conditions for extremal points in Eqn (6), by using the first derivative of $P$, which can be written as:

$$
\frac{d P}{d x}(x)=\pi(x) P(x),
$$

where the function $\pi$ is related to the fraction of inactive $D N A_{\text {off }}$ and $\rho$ (as given in Eqn (2)), which takes the form:

$$
\pi(x)=\frac{a(1-\varepsilon)}{x}[r(x)-\rho(x)]
$$


(A)
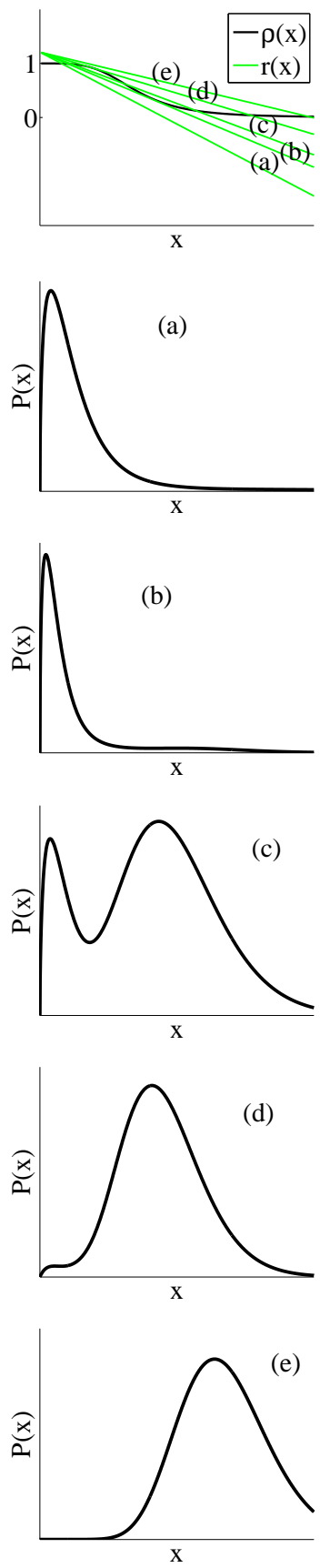

(B)

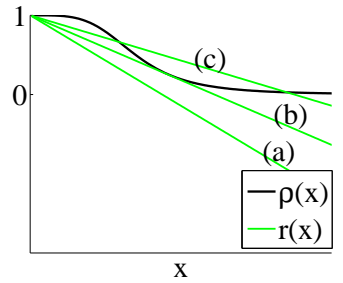

(a)

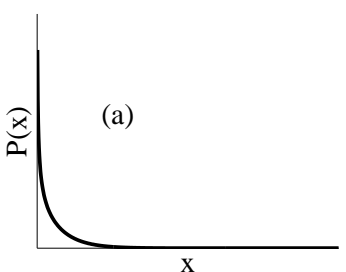

(b)
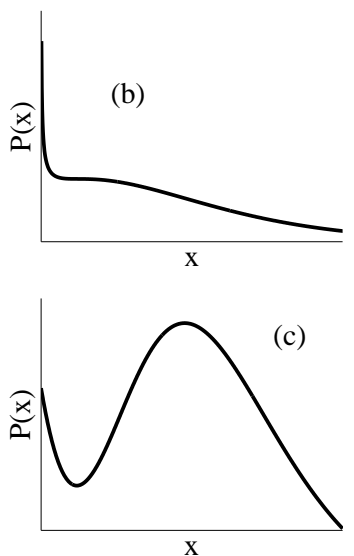

(C)

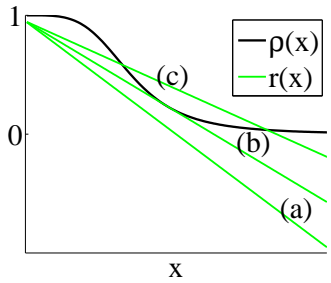

(a)

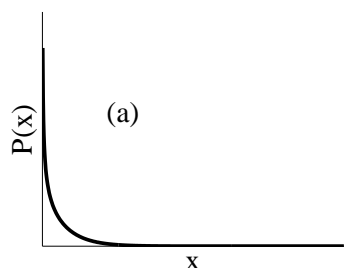

(b)

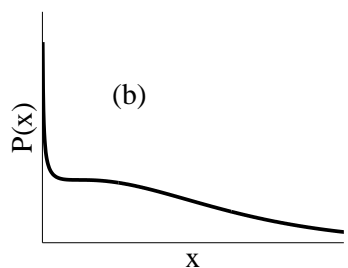

(c)

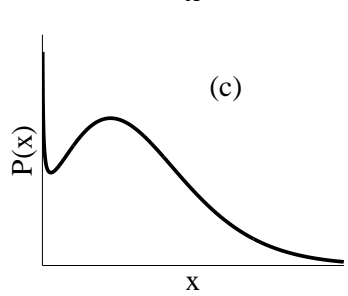

(D)

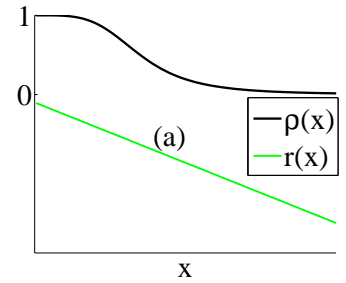

(a)

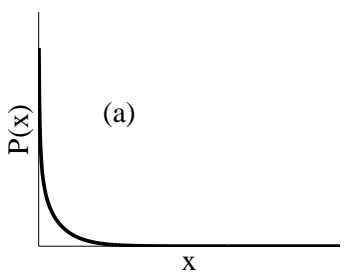

FIG. 4. The qualitative shape of all possible distributions for $H<-1$ depending on the intersections between $\rho(x)$ and $r(x)$ in Eqn (8) (black and green lines, respectively, on the first file plots). The different $r(x)$ are marked with a letter to identify which shape corresponds to each line. For $r(0)>1$ (row A), the number of intersection points ranges from one to three as the slope decreases, what corresponds with graded and bimodal distributions. For $0<r(0) \leq 1$ there can be at most two positive intersections that correspond with binary distributions (row B and C). For $r(0) \leq 0$ no intersection occurs (row D). (Color Online). 
with $r$ being the linear function

$$
r(x)=\frac{-x}{a b(1-\varepsilon)}+\frac{(a-1)}{a(1-\varepsilon)} .
$$

Since $P(x)>0$ for $x>0$, by using (8) the extremal points (i.e. points satisfying $\frac{d P}{d x}(x)=0$ ) are solutions of $\pi(x)=0$. Geometrically, extremal points are associated with the intersection of the graph of function $\rho$ with the straight line graph of $r$ (see also Figures 3 and 4). Formally, the set of extremal points is defined as:

$$
\mathcal{S}=\{x>0 \mid \rho(x)=r(x)\}
$$

Since the slope of $r(x)$ is always negative, the number of extremal points depends on the shape of $\rho(x)$ (Eqn (2)). This function turns out to depend on the class of self-regulating mechanism $H$. For negative feedback, the set $\mathcal{S}$ will contain at most one element. This follows directly from the fact that for $H>0$, the function $\rho$ is monotone increasing while the slope of the straight line graph of $r$ is negative for any set of parameters.

For positive feedback with $H=-1$, the set $\mathcal{S}$ contains at most two elements, whereas for $H<-1$, the number of elements in $\mathcal{S}$ is three at most (see Proposition A.2 in Appendix A for a formal proof). The intersection of Eqn (9) with the ordinate (i.e. the value $r(0)$ ), determines the exact number of extremal points as Figures 3 and 4 illustrate for positive feedback.

In this way, no point is expected if $r(0) \leq 0$ (i.e. $a \leq 1$ ), whereas for $0<r(0)<1$ there will be two at most. For $r(0)>1$, the maximum number will depend on $H$. In particular, for $H=-1$ it cannot be larger than two while under cooperativity $(H<-1)$ there will be three at most.

In order to identify the regions in the parameters space that support bimodal/binary behavior, it is not enough to know the number of intersections, but a functional dependence between parameters and distribution shape is needed. We derive such relationship from the condition for a minimum for $P(x)$, which will be the basis of the algorithm to compute the regions.

Maxima and minima in the set $\mathcal{S}$ are identified by checking the sign of the second derivative of $P$, which turns out to be of the form:

$$
\frac{d^{2} P}{d x^{2}}(x)=\left[\pi^{\prime}(x)+\pi^{2}(x)\right] P(x) .
$$


For any $x \in \mathcal{S}$ we have $\pi(x)=0$, so that the sign of the above expression is given by the sign of $\pi^{\prime}(x)$. This first derivative reads:

$$
\pi^{\prime}(x)=-\frac{\pi(x)}{x}+\frac{a(1-\varepsilon)}{x}\left[r^{\prime}(x)-\rho^{\prime}(x)\right] .
$$

By using the previous expression, the condition for an element $x \in \mathcal{S}$ to be a minimum (i.e. $\pi^{\prime}(x)>0$ ), and therefore having a bimodal/binary distribution, becomes:

$$
\rho^{\prime}(x)+\frac{1}{a b(1-\varepsilon)}<0 .
$$

Note that the corresponding condition for a maximum requires the above inequality to be reversed so that $\rho^{\prime}(x)-r^{\prime}(x)>0$.

For $H>0$ we have that $\rho^{\prime}(x)>0$ for $x>0$, so that condition $\rho^{\prime}(x)+1 /(a b(1-\varepsilon))>0$ holds everywhere. Therefore, according to (12), only graded distributions are expected since there can be at most one intersection point. For $H<0$ there is room for binary distributions, except when $r(0) \leq 0$, or for $H=-1$ when $r(0)>1$.

In general, bimodal/binary distributions require both inequality (12) for a minimum, and its converse for a maximum, to hold on complementary intervals within the positive real line.

For $H<-1$, let $x^{*}$ be such that the function $\rho^{\prime}$ attains a minimum, then $x^{*}$ can be obtained from equation $\rho^{\prime \prime}\left(x^{*}\right)=0$. Moreover, in the Appendix the expression of $x^{*}$ is given and we have:

$$
\rho^{\prime}\left(x^{*}\right)=\frac{H^{2}-1}{4 H x^{*}} .
$$

For any parameter set $(a, b$ and $\varepsilon)$ such that $-1 / a b(1-\varepsilon)$ lies between zero and $\rho^{\prime}\left(x^{*}\right)$ so that:

$$
\inf _{x} \rho^{\prime}(x)+\frac{1}{a b(1-\varepsilon)}<0,
$$

condition (12) holds for $x$ values in a interval $\left(\bar{x}_{1}, \bar{x}_{2}\right)$. Figure 5, which represent in solid lines a typical function $\rho(x)$ for $H<-1$ (upper plot) and its first derivative (lower plot), illustrates this situation.

Values $\bar{x}_{1}, \bar{x}_{2}$, and thus the intervals which contain the extremal points, can be computed for any set of parameters satisfying (14), as the roots of the equation:

$$
\rho^{\prime}(x)+\frac{1}{a b(1-\varepsilon)}=0 .
$$

For $H=-1, \inf _{x} \rho^{\prime}(x)=-1 / K$ is attained at $x=0$, thus Eqn (15) has only one root $\bar{x}_{2}$. Since from previous arguments, $\mathcal{S}$ contains at most two critical points for all parameter sets 
satisfying inequality (14), the distribution consists of a minimum in the interval $\left(0, \bar{x}_{2}\right)$, and a maximum in the interval $\left(\bar{x}_{2}, \infty\right)$.

For $H<-1$ (Figure 5), $\mathcal{S}$ contains a minimum in the interval $\left(\bar{x}_{1}, \bar{x}_{2}\right)$ and two maxima, one in each complementary intervals $\left(0, \bar{x}_{1}\right)$ and $\left(\bar{x}_{2}, \infty\right)$.

Note that while transitional dynamics are feasible for any possible positive feedback $(H \leq$ -1), bimodal distributions characterizing transitions between two protein levels demand cooperativity (i.e. $H<-1$ ), with parameters being such that the graph of $r$ intersects the graph of $\rho$ in three points (see Figure 4A, 4th plot from the left).
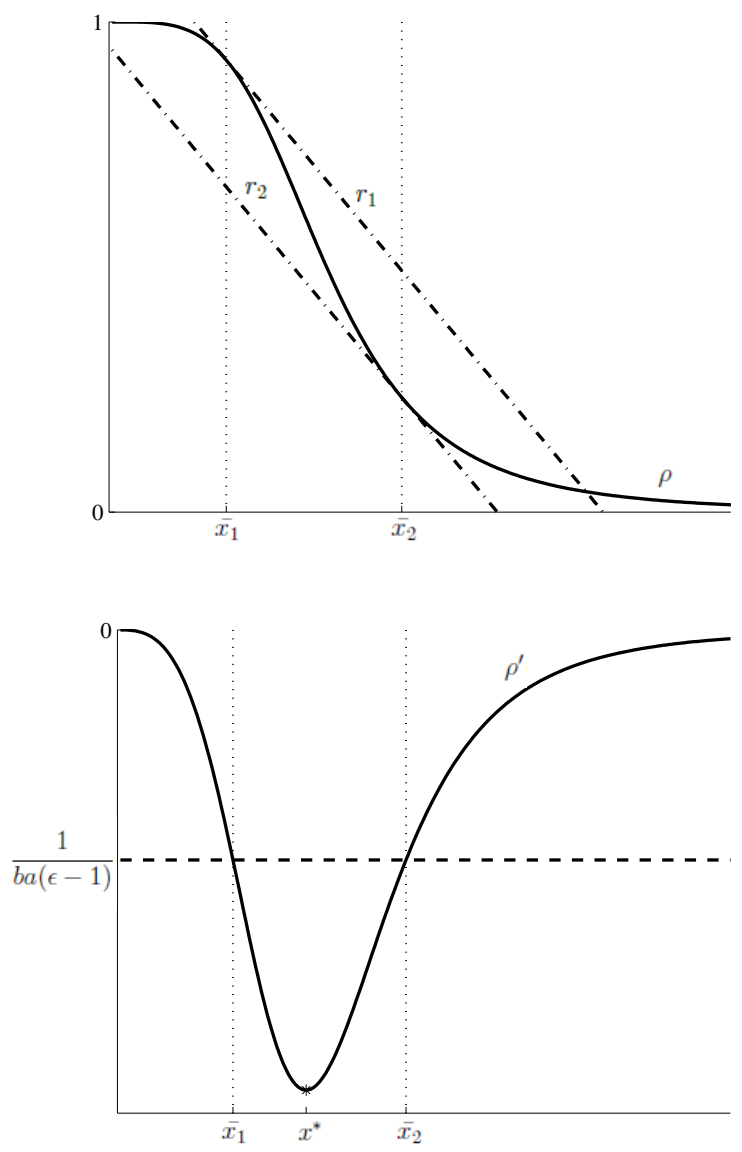

FIG. 5. The upper plot depicts function $\rho(x)$ as given in (2) for $H<-1$ and two possible functions $r_{1}(x)$ and $r_{2}(x)$ tangent to $\rho(x)$ at points $\left(\bar{x}_{1}\right.$ and $\left.\bar{x}_{2}\right)$, respectively. The lower plot represents the first derivative $\rho^{\prime}(x)$ and a possible value of $r^{\prime}(x)$ represented by the horizontal dashed line. Values $x \in \mathcal{S}$ which happen to be within the interval $\left(\bar{x}_{1}, \bar{x}_{2}\right)$ will satisfy condition (12) for a minimum since $\rho^{\prime}(x)<r^{\prime}(x)$. On the other hand, maxima will lie in intervals $\left(0, \bar{x}_{1}\right)$ and $\left(\bar{x}_{2}, \infty\right)$. 
Based on the above results that characterize analytically the minima and maxima, next we present necessary and sufficient conditions for binary/bimodal distributions and propose an algorithm to determine the region in the parameters space where such conditions are met.

\section{B. Necessary and sufficient conditions for bimodal/binary response}

In order to compute the parameter region that sustains transitional dynamics for cooperative networks (i.e. $H<-1$ ), first we need to obtain the values $\bar{x}_{1}$ and $\bar{x}_{2}$ which define the interval that should contain the minimum (thus satisfying (14) as previously discussed). For this purpose, we solve the following equation:

$$
\rho^{\prime}(x)-\sigma \rho^{\prime}\left(x^{*}\right)=0
$$

for every value $\sigma$ in the open interval $(0,1)$. Expression (16) combines Eqn (15) with the necessary condition (14). $\sigma$ in (16) can be interpreted as a sort of continuation parameter which goes through all possible values taken by $-1 / a b(1-\varepsilon)$ (ranging from zero to the minimum value of $\rho^{\prime}(x)$, provided by Eqn (13)) so to satisfy (14).

Taking into account that $\rho^{\prime \prime}(x) \neq 0$ for $x \neq x^{*}$, the implicit function theorem implies that the solutions $\bar{x}_{1}$ and $\bar{x}_{2}$ can be expressed as functions of $\sigma$ (for $0<x<x^{*}, \rho^{\prime \prime}(x)<0$, whereas for $\left.x^{*}<x<\infty, \rho^{\prime \prime}(x)>0\right)$. Let these functions be denoted by $\bar{x}_{1}=\varphi_{1}(\sigma)$ and $\bar{x}_{2}=\varphi_{2}(\sigma)$, and define two linear functions $r_{1}(x)$ and $r_{2}(x)$, the graph of which is tangent to the graph of $\rho$ at $\bar{x}_{1}$ and $\bar{x}_{2}$, respectively, so that:

$$
\begin{aligned}
& r_{1}(x)=\rho^{\prime}\left(\bar{x}_{1}\right)\left(x-\bar{x}_{1}\right)+\rho\left(\bar{x}_{1}\right), \\
& r_{2}(x)=\rho^{\prime}\left(\bar{x}_{2}\right)\left(x-\bar{x}_{2}\right)+\rho\left(\bar{x}_{2}\right) .
\end{aligned}
$$

By construction $r_{2}(x)<r(x)<r_{1}(x)$ for any $x$, so that the graph of $r$ intersects the one of $\rho$ at least in two points, one of them satisfying the condition for minimum. If in addition $r(0)>1$, the graph of $r$ intersects in exactly three points (see Proposition A.3): those in the intervals $\left(0, \bar{x}_{1}\right)$ and $\left(\bar{x}_{2}, \infty\right)$ being maxima, whereas the one in $\left(\bar{x}_{1}, \bar{x}_{2}\right)$ being a minimum. In this way, necessary and sufficient conditions for binary/bimodal distributions become:

$$
\begin{array}{ll}
\text { binary: } & r_{2}(x)<r(x)<r_{1}(x), \quad r(0) \leq 1 \\
\text { bimodal: } & r_{2}(x)<r(x)<r_{1}(x), r(0)>1
\end{array}
$$


From these inequalities, and using the fact that because of (15):

$$
\rho^{\prime}\left(\bar{x}_{1}\right)=\rho^{\prime}\left(\bar{x}_{2}\right) \equiv-\frac{1}{a b(1-\varepsilon)},
$$

we get, after reordering terms, the following constraints in the parameters space:

$$
\begin{gathered}
a>\frac{1}{\varepsilon} \\
\mathrm{h}\left(\bar{x}_{2} ; b\right)<a<\mathrm{h}\left(\bar{x}_{1} ; b\right)
\end{gathered}
$$

where $\mathrm{h}(x ; b)=\frac{b+x}{b \bar{c}(x)}$ and $\bar{c}(x)$ is given by expression (3). Inequality conditions (18-19) define the region of transitional dynamics in the parameters space, also including bimodal distributions associated to transitions between two most frequent positive states. The algorithm employed to compute the region is summarized in Table II.

TABLE II. Algorithm to find the region that corresponds with binary/bimodal distributions in the parameters space

To fix $H, K, \varepsilon$

$$
\begin{aligned}
& x^{*} \leftarrow \rho^{\prime}\left(x^{*}\right)=\min \rho^{\prime}(x) \\
& \text { for } \sigma \in\left(\begin{array}{ll}
0 & 1
\end{array}\right) \text { do } \\
& \delta^{*}=\sigma \rho^{\prime}\left(x^{*}\right) \\
& \overline{x_{1}}, \overline{x_{2}} \leftarrow \rho^{\prime}\left(\overline{x_{1}}\right)=\rho^{\prime}\left(\overline{x_{2}}\right)=\delta^{*} \\
& \varphi_{1}(\sigma)=\overline{x_{1}} \\
& \varphi_{2}(\sigma)=\overline{x_{2}}
\end{aligned}
$$

for $b>0$ do

To calculate $\mathrm{h}\left(\bar{x}_{1} ; b\right), \mathrm{h}\left(\bar{x}_{2} ; b\right)$

$A_{\sigma}=(a, b)$ such that $\mathrm{h}\left(\bar{x}_{2} ; b\right)<a<\mathrm{h}\left(\bar{x}_{1} ; b\right)$

\section{end for}

\section{end for}

$A=\cup_{\sigma} A_{\sigma}$

\section{RESULTS AND DISCUSSION}

The methods discussed in Section III for the self-regulated gene expression network previously described in Section II, are employed to explore the parameters space in search for 
regions sustaining a binary/bimodal distribution. One main result from our analysis concerns with the shape that the regions will exhibit in the space defined by the transcription and translation rate constants, $a$ and $b$, respectively. Regions linked to binary/bimodal distributions may appear as a stripped band that asymptotically approaches the $a$ and $b$ axes, thus splitting the parameters space. Alternatively, horn-like regions may result from the intersection of the upper and lower constraints in (19). Figures $6 \mathrm{~A}$ and $6 \mathrm{~B}$ show examples of such regions, computed with the algorithm summarized in Table II.

Remarkably, leakage level is the factor that determines shape. In this way, as leakage increases the band becomes narrower up to reach a point where the upper and lower bounds in (19) touch each other, and the horn-like region emerges. In fact, a critical leakage $\varepsilon^{*}$ has been identified which determines the transition from strip-like to horn-like regions. More precisely, we have:

$$
\begin{array}{lll}
\varepsilon>\varepsilon^{*} & \Leftrightarrow & \text { horn-like region } \\
\varepsilon \leq \varepsilon^{*} & \Leftrightarrow & \text { strip-like region }
\end{array}
$$

The parameter $\varepsilon^{*}$ can be calculated from the distance between the upper and lower bounds in expression (19) as it is detailed in Appendix B. Moreover, this parameter only depends on the level of cooperativity, namely the number of binding sites needed to activate the promoter, and takes the form:

$$
\varepsilon^{*}=\left(\frac{H+1}{H-1}\right)^{2} .
$$

In constructing the regions (in the $a-b$ parameter space) that support bimodal/binary distributions, a quantitative indicator of the reliability of the approximation is required. In this study, the relative error between the analytic solution and the corresponding to the stationary CME has been employed.

Figure 6A presents a contour plot of the relative error for a strip-like region. As the figure shows, divergences essentially occur for small bursts (i.e. low b), usually located near the lower bound of the region, where a relative error around a $4 \%$ is observed. Nonetheless, the agreement between CME and the approximation (6) is in general quite acceptable for most part of the parameters space, what includes the lower bound of the region.

As an example: for the point marked in the region in Figure 6A, which coincides with a high (around a 4\%) relative error, the analytical distribution is in a quite reasonable agreement with the distribution computed from the solution of the CME (Figure 6C). The 
approximation becomes even better for the horn-like region since this one corresponds with larger burst sizes. Figure 6D illustrates the excellent agreement found between the analytical solution and the distribution obtained from the CME, for a parameter set marked as a dot in Figure 6B. Figures 6E and 6F depict the corresponding stochastic dynamics.

The evolution in Figure 6E shows a typical switching with regular but infrequent transitions between low and high protein states. Such behavior is in correspondence with the distribution depicted in Figure 6C, which presents a very low probability valley separating both (maximum frequency) protein levels. Its dynamics contrasts with the one observed in Figure $6 \mathrm{~F}$, where the two most frequent states indicated in the corresponding distribution (plot D) are much more frequently visited, thus leading to a quite noisy response.

One might argue that a standard analysis based on a deterministic description of the selfregulatory network may offer a more amenable route to study bimodal/binary responses in stochastic systems. Unfortunately, despite some situations in which a bistable deterministic counterpart may approximate a stochastic system showing bimodality, this approach can be misleading. In order to understand this point, let us consider the deterministic counterpart of the system depicted in Figure 1, which can be written as:

$$
\begin{aligned}
\frac{\mathrm{d} m}{\mathrm{~d} \tau} & =a \bar{c}(x)-\frac{\gamma_{1}}{\gamma_{2}} m \\
\frac{\mathrm{d} x}{\mathrm{~d} \tau} & =b \frac{\gamma_{1}}{\gamma_{2}} m-x
\end{aligned}
$$

where $m$ and $x$ represent the concentrations of $m R N A$ and protein, respectively, and $\tau=\gamma_{2} t$ is the dimensionless time associated, as in Eqn (4), to the scale of protein degradation. From the steady state solution (satisfying $\mathrm{d} m / \mathrm{d} \tau=\mathrm{d} x / \mathrm{d} \tau=0$ ), we have that:

$$
m(x)=a \frac{\gamma_{2}}{\gamma_{1}} \bar{c}(x)
$$

Replacing the above expression in (22), using Eqn (3) and re-ordering terms, we finally obtain the equilibrium states for the system at the roots of the equation $r_{d}(x)-\rho(x)=0$, where:

$$
r_{d}(x):=\frac{-x}{a b(1-\varepsilon)}+\frac{1}{1-\varepsilon} .
$$

Interestingly, the above expression derived from the deterministic analysis is similar to Eqn (9), but by no means equivalent. Both expressions (9) and (24) share the same slope, but their values at zero are different. Consequently, as illustrated in Figure 7, a shift between 
$r(x)$ and $r_{d}(x)$ is produced what explains the existence of bimodality on a stochastic system and monostability on its deterministic counterpart. Figure 8 presents the corresponding bimodal distribution together with a realization of the stochastic dynamics. The trajectories exhibited by the deterministic system are presented in Figure 9 for two sets of parameters, one corresponding to a monostable equilibrium (Figure 9A) and the other one associated with a bistable system (Figure 9B).

The algorithm in Table II has been employed to compute bimodal/binary regions for different feedback strengths and leakage levels. The influence of the (positive) feedback on the extensions of the regions is given by the so-called feedback strength $c=K^{-|H|}([31])$, and the level of basal expression represented by the leakage factor $\varepsilon$, which appears in (3). Note that binary/bimodal responses persist for positive feedback with $H<-1$ under quite different conditions. However, the region withdraws to areas of low transcription and high translation rates as leakage crosses the critical value $\varepsilon^{*}$ given by expression (20). Figure 10 illustrates the influence of network parameters on the shape of the binary/bimodal regions.

Either because the number of protein molecules needed to activate $D N A$ increases (so that $H$ becomes more negative) or because the ratio of inactive versus active forms $(K)$ becomes higher, the region expands as the feedback strength decreases for a given leakage level. On the other hand, for a given feedback strength, increasing the leakage level reduces region's width as illustrated in Figure $10 \mathrm{C}$.

Figure 10 also includes the parameter regions that lead the deterministic system to bistability. Such regions (bounded by dashed lines) partially overlap the corresponding stochastic ones sustaining bimodal/binary distributions. However, note that the bistable behavior disappears above the critical leakage $\varepsilon^{*}$, thus never co-existing with bimodal/binary horn-like regions.

\section{CONCLUSIONS}

In this work, we have proposed a methodology to characterize the shape of stationary protein distributions for self-regulatory stochastic gene expression networks. The method defines regions in the parameters space capable of sustaining bimodal/binary protein distributions, in the understanding that such distributions are indicative of transitional dynamics switching between two most frequent states. 
(A)

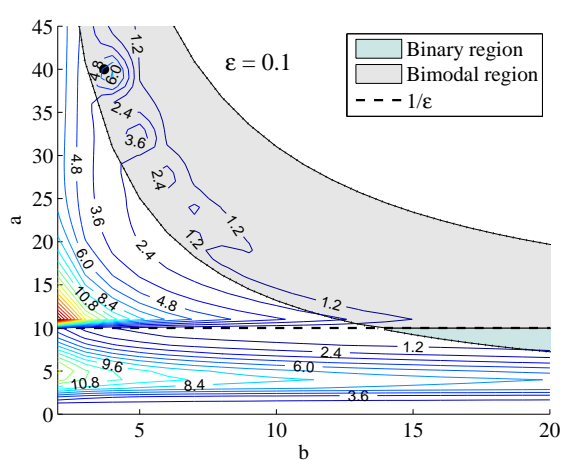

$(\mathrm{C})$

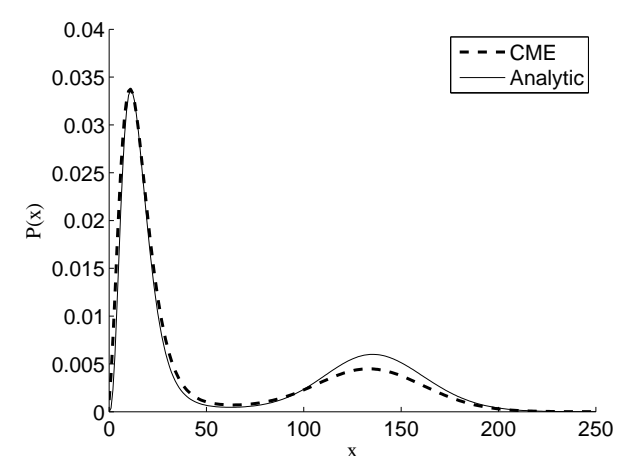

$(\mathrm{E})$

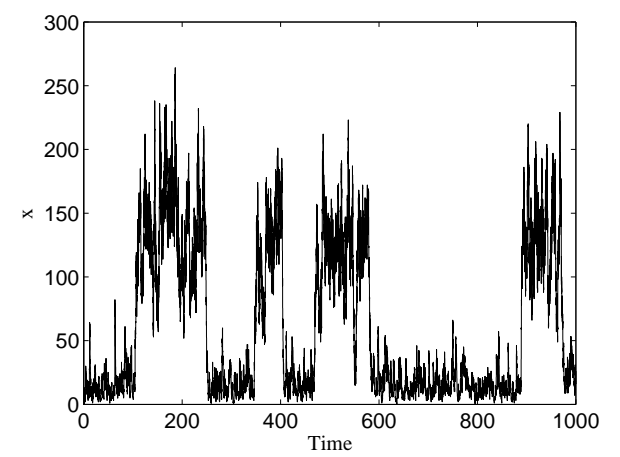

(B)

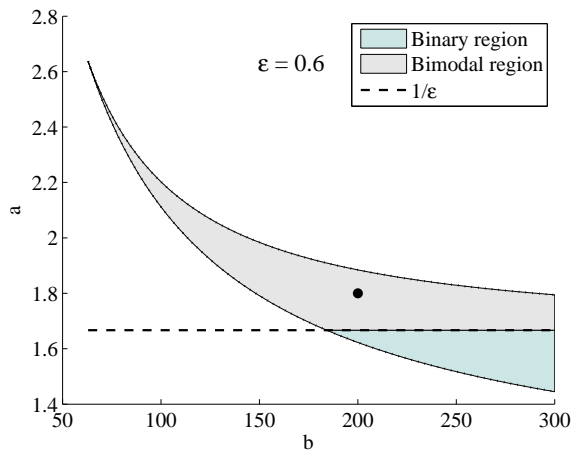

(D)

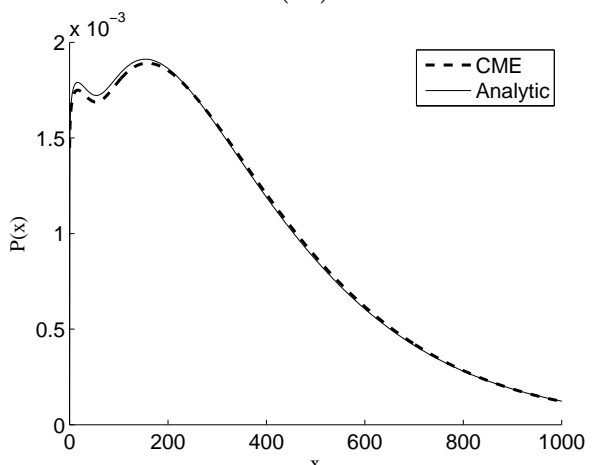

$(\mathrm{F})$

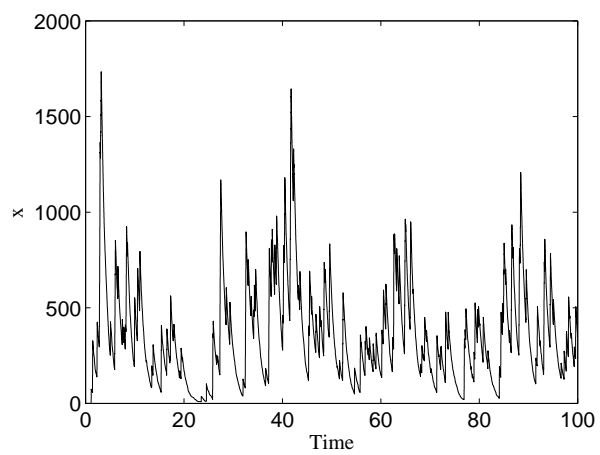

FIG. 6. Possible shapes of the binary/bimodal regions for $K=70$ and $H=-4$ (according to expression (20), $\varepsilon^{*}=0.36$ for these parameter values). (A) Strip-like region corresponding to $\varepsilon<\varepsilon^{*}$. (B) Horn-like region corresponding to $\varepsilon>\varepsilon^{*}$. Contours in plot A provide an indication of the relative error between the analytical distribution and the distribution computed from the CME. For most of the region the error remains quite low, rising to about $4 \%$ only near the lower bound of the region. Plots $\mathrm{C}$ and $\mathrm{D}$ represent the stationary distributions associated to the points marked in the corresponding regions (A and B, respectively). For comparison purposes, distributions are computed from the analytical solution, Eqn (6), as well as from the CME. Plots E and F depict the corresponding stochastic dynamics (SSA). Plot E reflects a scenario with rare transitions between high and low protein levels, while plot $\mathrm{F}$ represents a noisy behavior with frequent transitions between high and low protein levels. (Color Online). 
Unlike other approaches using a deterministic description, the one here presented is based on the continuous approximation proposed by [29] for CME. Thus, the proposed method allows the identification of transitional dynamics associated to bimodal distributions which otherwise would correspond with deterministic monostable systems.

Results reveal that bimodal behavior is a ubiquitous phenomenon in cooperative gene expression networks under positive feedback. It is present for any range of transcription and translation rate constants whenever leakage remains below a critical threshold. Above such threshold, the region in the space of parameters which sustains bimodality persists, although withdrawn to low transcription and high translation rate constants. Remarkably, such threshold is independent of the transcription/translation rates or the proportion of active/inactive promoter, depending only on the level of cooperativity. The method presented here might be of help in the context of synthetic biology to guide tuning of self-regulatory networks with specific functionalities.

From the point of view of designing gene circuits, it may be of interest to search for a

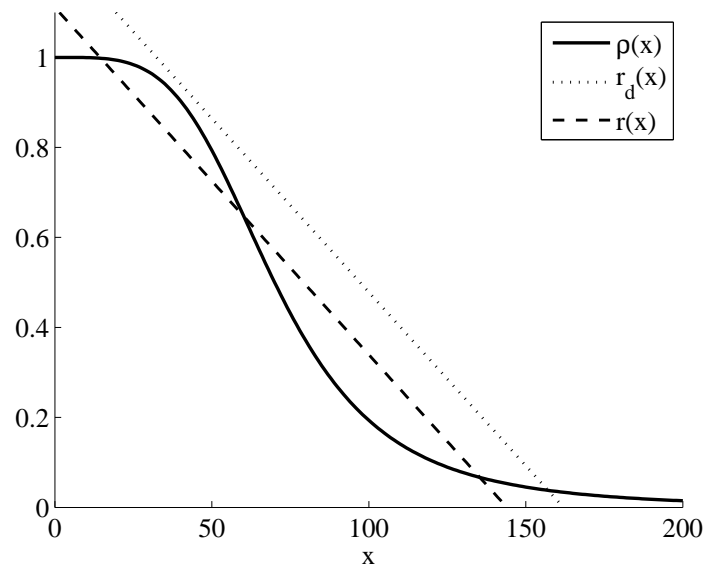

FIG. 7. Intersections of $\rho(x)$ (continuous line) with $r(x)$ (Eqn (9)) associated to stochastic analysis (dashed line), and $r_{d}(x)$ (Eqn (24)) associated to deterministic ODEs (dotted line), for a selfregulatory network with parameters $H=-4, K=70, \varepsilon=0.2, a=9, b=18 . r_{d}(x)$ is shifted with respect to $r(x)$, what leads to one intersection point instead of three. Consequently, the deterministic system shows one single (monostable) equilibrium state whereas the stochastic counterpart has a bimodal distribution compatible with a transitional dynamics, switching between two positive states. 
particular distribution with some characteristic average levels for protein, certain limits in the admissible noise level or frequency of the transitions between states, for instance. In addition, the framework we present can be adapted to undertake more complex circuit design problems. For instance, by formulating them in the context of optimization problems with the objectives oriented to produce bimodal/binary responses minimizing noise or switching transition frequency.

Acknowledgements This work has been partially supported by Ministerio de Economia y Competitividad AGL2012-39951-C02-01. M.P. acknowledge support from Grant No. BES-
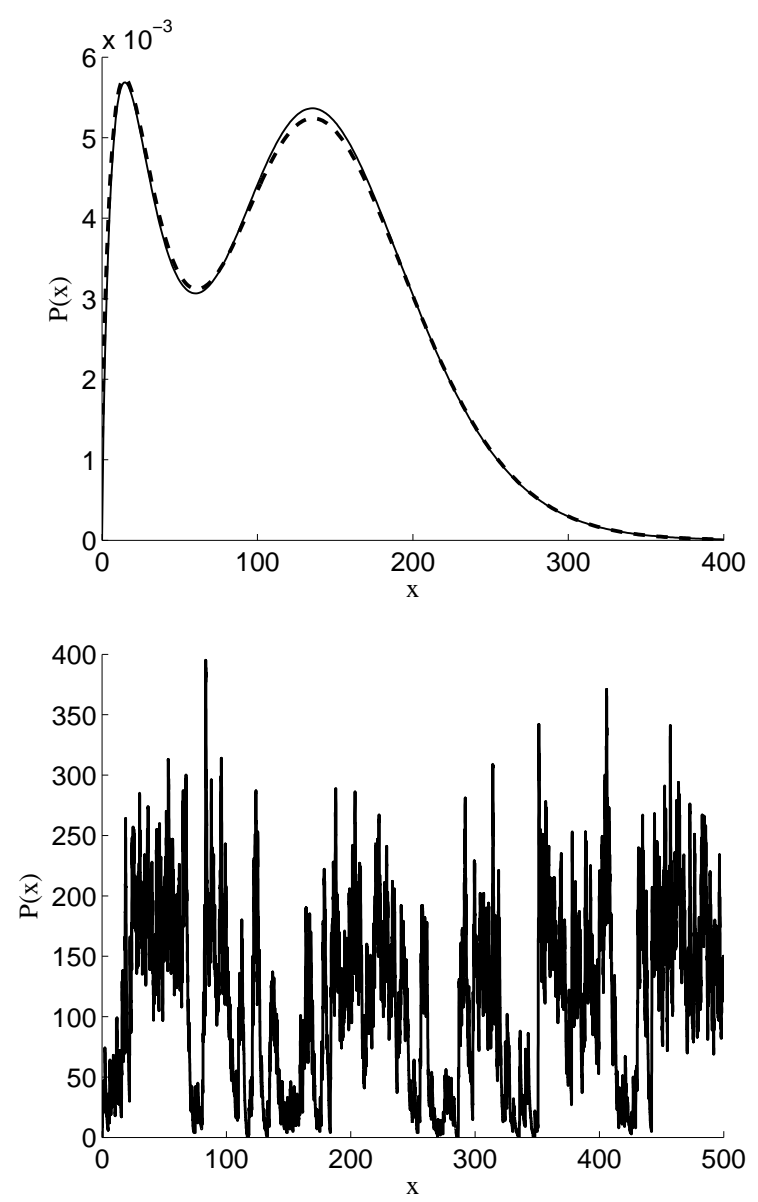

FIG. 8. A self-regulatory network with parameters $H=-4, K=70, \varepsilon=0.2, a=9, b=18$. (A) Stationary distribution computed from the CME (dashed line) and the analytical distribution (continuous line). The two most frequent states in the distribution correspond with the intersections between $\rho(x)$ and $r(x)$ shown in Figure (7). (B) The corresponding stochastic dynamics (SSA) with regular transitions taking place between a low and a high protein level. 
(A)

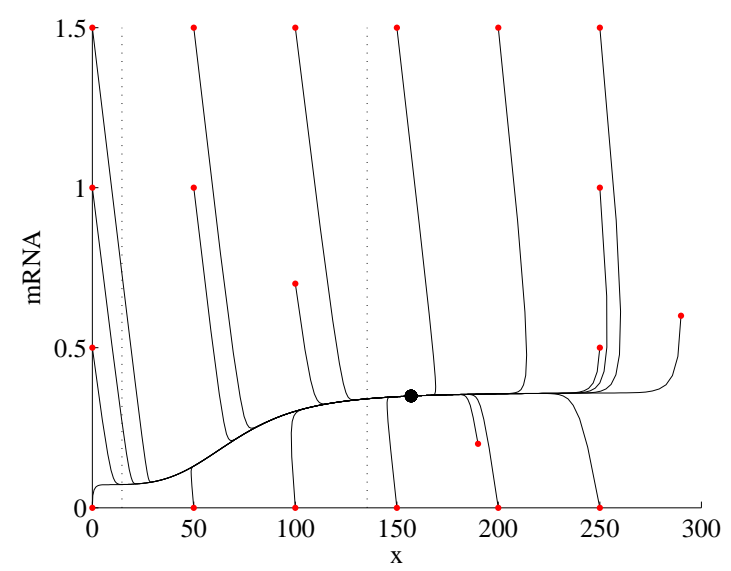

(B)

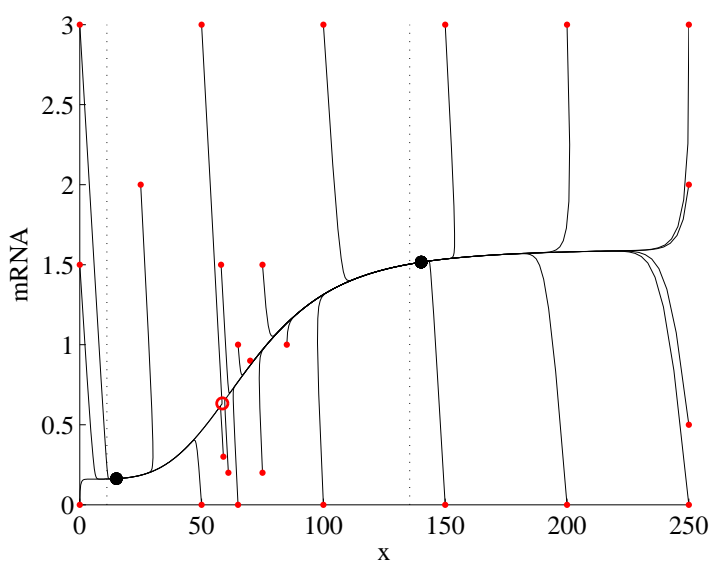

FIG. 9. Trajectories exhibited by the corresponding deterministic system. (A) Trajectories associated to a network with parameters $H=-4, K=70, \varepsilon=0.2, a=9, b=18$ (see also Figure 8) evolving to a stable equilibrium point. (B) Trajectories associated to a network with parameters $H=-4, K=70, \varepsilon=0.1, a=40, b=3.7$. Red circle and black dots correspond with the unstable and stable equilibrium points, respectively. (Color Online).

2013-063112. C.V. has been partially supported by MTM2013-47800-C2-1-P.

\section{Appendix A: Characterizing the stationary distributions}

This Appendix gives some results including the analytical steady state solution associated to Equation (4), and a formal characterization of the extremal points associated to it which turns out to correspond with the stationary protein distribution.

Proposition A.1 The stationary solution of equation (4) is given by

$$
P(x)=C\left(K^{H}+x^{H}\right)^{\frac{a(\varepsilon-1)}{H}} x^{a-1} e^{\frac{-x}{b}}, \quad x \in(0 \infty),
$$

where $C$ is the integration constant that guarantees condition $\int_{0}^{\infty} p(x) \mathrm{d} x=1$, and the parameters $H, K, \varepsilon, a=\frac{k_{1}}{\gamma_{2}}$ and $b=\frac{k_{2}}{\gamma_{1}}$ are those in equation (4).

Proof: Let $P$ denote the stationary solution (which only depends on $x$ ) to make the difference with the evolutive solution $p$ (depending on $\tau$ and $x$ ). First, in order to obtain the equation for the steady state solution we consider Equation (4) with $\frac{\partial p(\tau, x)}{\partial \tau}=0$, so 
(A)
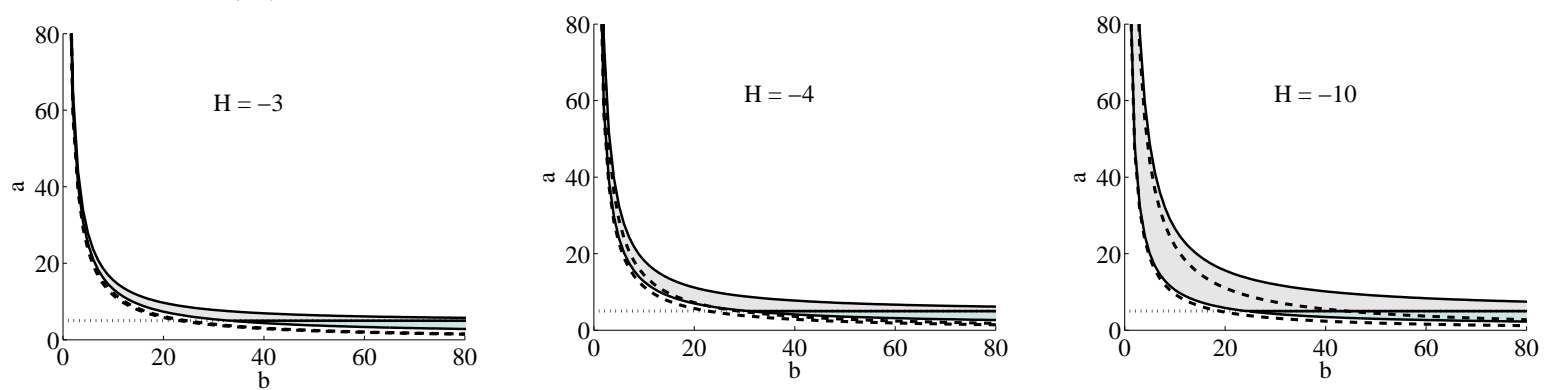

(B)
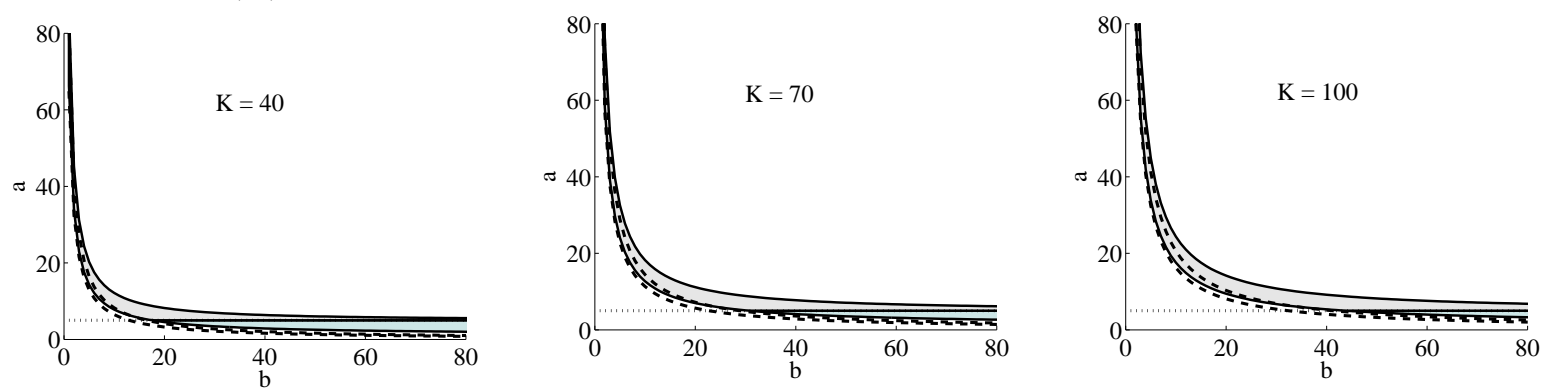

(C)
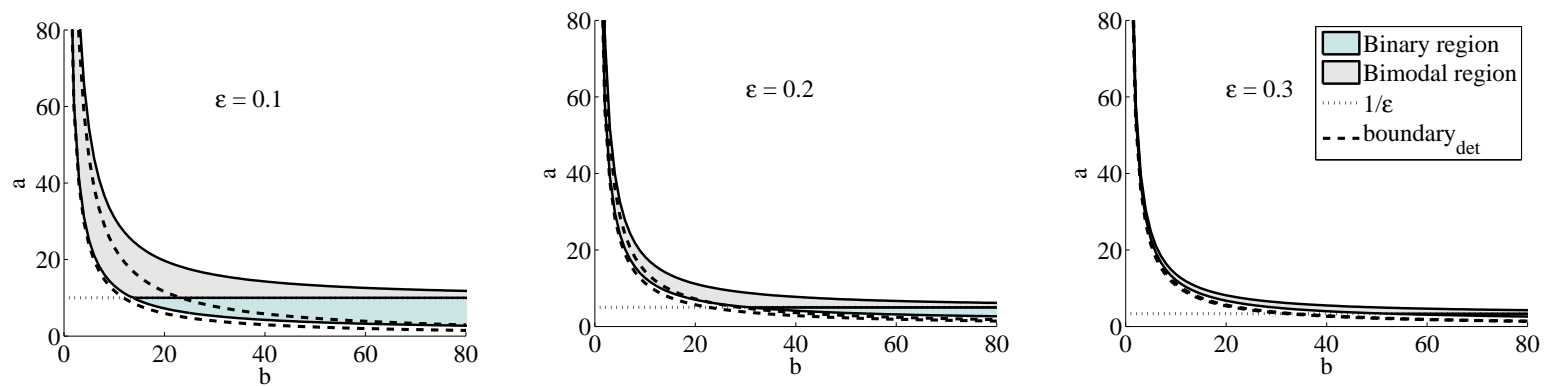

FIG. 10. Regions sustaining bimodal/binary distributions in the parameters space. Regions in the $a-b$ parameter space reduce to the strip shaded area. Areas in grey (shaded region 2) denote regions with bimodal distributions. Dashed lines represent the bistable region boundaries of the deterministic model. (A) Effect of $H$ on the region area for $K=70$, and $\varepsilon=0.2$, below the critical value $\varepsilon^{*}=0.25$ (for $H=-3$ ), $\varepsilon^{*}=0.36$ (for $H=-4$ ) and $\varepsilon^{*}=0.6694$ (for $H=-10$ ). (B) Effect of $K$ on the region area, with $K=40, K=70$ and $K=100$, for $\varepsilon=0.2$ and $H=-4$. (C) Effect of $\varepsilon$ on the region area, with $\varepsilon=0.1, \varepsilon=0.2$ and $\varepsilon=0.3$, for $K=70$ and $H=-4$.

that $P$ satisfies

$$
Z(P)(x)=\frac{d}{d x}\left(\gamma_{2} x P(x)\right)+\frac{k_{1}}{b} \int_{0}^{x} \exp \left(\frac{x^{\prime}-x}{b}\right) \bar{c}\left(x^{\prime}\right) P\left(x^{\prime}\right) \mathrm{d} x^{\prime}-k_{1} \bar{c}(x) P(x)=0 .
$$

Next, after multiplying (A1) by $\exp (x / b)$ and taking derivative with respect to $x$, we get

$$
\frac{d}{d x}\left[e^{\frac{x}{b}}\left(-\frac{d}{d x}\left(\gamma_{2} x P(x)\right)+k_{1} \bar{c}(x) P(x)\right)\right]=\frac{d}{d x}\left[\frac{k_{1}}{b} \int_{0}^{x} e^{\frac{x^{\prime}}{b}} \bar{c}\left(x^{\prime}\right) P\left(x^{\prime}\right) \mathrm{d} x^{\prime}\right] .
$$


Next, applying the classical formula

$$
\frac{d}{d x} \int_{a(x)}^{b(x)} f\left(x^{\prime}\right) \mathrm{d} x^{\prime}=f(b(x)) \frac{d b}{d x}(x)-f(a(x)) \frac{d a}{d x}(x),
$$

we can eliminate the integral term in (A4) to obtain

$$
\frac{d}{d x}\left[e^{\frac{x}{b}}\left(-\frac{d}{d x}\left(\gamma_{2} x P(x)\right)+k_{1} \bar{c}(x) P(x)\right)\right]=\frac{k_{1}}{b} e^{\frac{x}{b}} \bar{c}(x) P(x) .
$$

After some computations in (A4) we get the following homogeneous linear second order ODE;

$$
A_{2}(x) \frac{d P}{d x^{2}}+A_{1}(x) \frac{d P}{d x}+A_{0}(x) P=0
$$

with variable coefficients

$$
A_{2}(x)=-\gamma_{2} x, \quad A_{1}(x)=k_{1} \bar{c}(x)-2 \gamma_{2}-\frac{\gamma_{2} x}{b}, \quad A_{0}(x)=k_{1} \frac{d \bar{c}}{d x}(x)-\frac{\gamma_{2}}{b} .
$$

Note that the coefficients in (A5) satisfy condition $A_{2}^{\prime \prime}-A_{1}^{\prime}+A_{0}=0$, which is equivalent to (A5) being an exact ODE (see [39], for example). In fact, Equation (A5) can be written in equivalent form as

$$
\frac{d}{d x}\left[A(x) \frac{d P}{d x}+B(x) P\right]=0
$$

where $A(x)=A_{2}(x)=-\gamma_{2} x$ and $B(x)=A_{1}(x)-A_{2}^{\prime}=k_{1} \bar{c}(x)-\gamma_{2}-\frac{\gamma_{2} x}{b}$. Next, by integrating (A6) we get

$$
A(x) \frac{\partial P(x)}{\partial x}+B(x) P(x)=K_{c}
$$

where $K_{c}$ is a constant. Moreover, we can choose $K_{c}=0$. This can be justified since $Z(P)(x)=0$ implies that $-Z(P)(x)=Z(P)(x)$. Then, $K_{c}=0$ if and only if

$$
A(x) \frac{d P}{d x}(x)+B(x) P(x)=-Z(P)(x)=0=k_{c} .
$$

Then $A(x) \frac{d P}{d x}(x)+B(x) P(x)=-Z(P)(x) \Leftrightarrow e^{\frac{x}{b}} x P(x)=a \int_{0}^{x} e^{\frac{x^{\prime}}{b}} \bar{c}\left(x^{\prime}\right) P\left(x^{\prime}\right) \mathrm{d} x^{\prime} \Leftrightarrow \frac{d}{d x}\left[e^{\frac{x}{b}} x P(x)\right]=$ $\frac{d}{d x}\left[a \int_{0}^{x} e^{\frac{x^{\prime}}{b}} \bar{c}\left(x^{\prime}\right) P\left(x^{\prime}\right) \mathrm{d} x^{\prime}\right] \Leftrightarrow x \frac{d P}{d x}(x)=\left[a \bar{c}(x)-\frac{x}{b}-1\right] P(x) \Leftrightarrow \frac{d P}{d x}(x)=\left[\frac{a \bar{c}(x)}{x}-\frac{1}{b}-\frac{1}{x}\right] P(x)$.

Finally $\frac{a \bar{c}(x)}{x}-\frac{1}{b}-\frac{1}{x}=\frac{-B(x)}{A(x)}$ and thus we prove that $K_{c}=0$. Then we get the first order linear ODE

$$
\frac{d P}{d x}(x)=\frac{-B(x)}{A(x)} P(x),
$$


where $\frac{-B(x)}{A(x)}$ is defined for $x \in(0 \infty)$. So, we have the solution

$$
P(x)=C \exp \left(\int \frac{-B(x)}{A(x)} \mathrm{d} x\right), \quad x \in(0 \infty),
$$

where

$$
\begin{aligned}
\int \frac{-B(x)}{A(x)} \mathrm{d} x & =\int\left(\frac{a \bar{c}(x)}{x}-\frac{1}{x}-\frac{1}{b}\right) \mathrm{d} x \\
& =\int\left(\frac{a K^{H}}{\left(K^{H}+x^{H}\right) x}+\frac{a \varepsilon x^{H-1}}{K^{H}+x^{H}}-\frac{1}{x}-\frac{1}{b}\right) \mathrm{d} x \\
& =\int\left(a(\varepsilon-1) \frac{x^{H-1}}{K^{H}+x^{H}}+\frac{a-1}{x}-\frac{1}{b}\right) \mathrm{d} x \\
& =\frac{a(\varepsilon-1)}{H} \ln \left(K^{H}+x^{H}\right)+(a-1) \ln (x)-\frac{x}{b} .
\end{aligned}
$$

Thus, the stationary solution is given by

$$
P(x)=C\left(K^{H}+x^{H}\right)^{\frac{a(\varepsilon-1)}{H}} x^{a-1} e^{\frac{-x}{b}}, x \in(0 \infty)
$$

where $C$ is a integration constant imposing that $\int_{0}^{\infty} p(x) \mathrm{d} x=1$.

Proposition A.2 For $H=-1$, the set $\mathcal{S}$ contains at most two elements.

For $H<-1$, the set $\mathcal{S}$ contains at most three elements.

Proof: It is a standard result that any straight line can intersect the graph of a convex function $f: \mathbb{R} \rightarrow \mathbb{R}$ at most in two points. For any convex function $f: \mathbb{R} \rightarrow \mathbb{R}$ and any point $x \in\left[x_{1}, x_{2}\right]$ the inequality $\mathrm{f}(x)<(1-\lambda) \mathrm{f}\left(x_{1}\right)+\lambda \mathrm{f}\left(x_{2}\right)$ is satisfied for any $\lambda \in(0,1)$. So, by choosing $\lambda=\left(x-x_{1}\right) /\left(x_{2}-x_{1}\right)$ the straight line graph of $y(x)=\mathrm{f}\left(x_{1}\right)+\frac{\mathrm{f}\left(x_{2}\right)-\mathrm{f}\left(x_{1}\right)}{x_{2}-x_{1}}\left(x-x_{1}\right)$ intersects the graph of $\mathrm{f}$ in at most two points $\left(x_{1}\right.$ and $\left.x_{2}\right)$. We make use of this fact to prove the statements for the function $\rho$ over its domain $[0, \infty)$. In order to study convexity of $\rho$ we make use of its second derivative, given as

$$
\rho^{\prime \prime}(x)=\frac{H}{x^{2}} \rho(x)(1-\rho(x))[H(1-2 \rho(x))-1],
$$

which for $H=-1$ is strictly positive for $x>0$, thus proving the first statement.

For $H<-1, \rho$ it can be proved that $\rho^{\prime \prime}\left(x^{*}\right)=0$ for $x^{*}=K \sqrt[H]{\frac{H-1}{H+1}}$, so that $\rho$ is concave (i.e $\rho^{\prime \prime}(x)<0$ ) for $x \leq x^{*}$. Moreover

$$
\rho\left(x^{*}\right)=(H-1) / 2 H
$$

Since it is concave (i.e $\left.\rho^{\prime \prime}(x)<0\right)$ for $x \in\left(0, x^{*}\right)$ and convex in $\left(x^{*}, \infty\right)$, the graph of $r$ intersects the graph of $\rho$ in three points at most, what concludes the proof of the second statement. 
Proposition A.3 The maximum number of extremal points for the stationary solution (4) is:

1. None if $r(0) \leq 0$ (i.e. $a \leq 1)$.

2. Two at most if $0<r(0)<1$ (i.e. $1<a<\varepsilon^{-1}$ ).

3. Two at most (one at $x=0$ ) if $r(0)=1$ (i.e. $a=\varepsilon^{-1}$ ), and $H=-1$.

4. Three at most (one at $x=0$ ) if $r(0)=1$ (i.e. $a=\varepsilon^{-1}$ ), and $H<-1$.

5. Exactly one if $r(0)>1$ (i.e. $a>\varepsilon^{-1}$ ), and $H=-1$.

6. At least one and at most three if $r(0)>1$ (i.e. $a>\varepsilon^{-1}$ ), and $H<-1$.

Proof: The different statements can be proved in a straightforward manner by checking the intersections and noting that $r^{\prime}(x)=-(a b(1-\varepsilon))^{-1}<0$ (a negative constant) for any admissible set of parameters and the properties of $\rho$ and its derivatives (for illustrative purposes, see also Figures 3 and 4).

\section{Appendix B: Computing a critical leakage factor $\varepsilon^{*}$}

The distance between the bounds $h\left(\bar{x}_{2} ; b\right)$ and $h\left(\bar{x}_{1} ; b\right)$ in $(19)$ constraints can be expressed as:

$$
\triangle \mathrm{h}(\sigma, b)=\frac{\bar{c}\left(\bar{x}_{2}\right)-\bar{c}\left(\bar{x}_{1}\right)}{\bar{c}\left(\bar{x}_{1}\right) \bar{c}\left(\bar{x}_{2}\right)}\left(1-\frac{b^{*}(\sigma)}{b}\right)
$$

where $b^{*}(\sigma)$ is given by

$$
b^{*}(\sigma)=\frac{\bar{x}_{2} \bar{c}\left(\bar{x}_{1}\right)-\bar{x}_{1} \bar{c}\left(\bar{x}_{2}\right)}{\bar{c}\left(\bar{x}_{2}\right)-\bar{c}\left(\bar{x}_{1}\right)} \equiv \frac{\bar{x}_{2}-\bar{x}_{1}-(1-\varepsilon)\left(\rho\left(\bar{x}_{1}\right) \bar{x}_{2}-\rho\left(\bar{x}_{2}\right) \bar{x}_{1}\right)}{(1-\varepsilon)\left(\rho\left(\bar{x}_{1}\right)-\rho\left(\bar{x}_{2}\right)\right)}
$$

The horn-like region appears whenever both bounds coincide, i.e. when $\Delta \mathrm{h}(\sigma, b)=0$, for all $\sigma \in\left(\begin{array}{ll}0 & 1\end{array}\right)$ and $b>0$. This is equivalent to $b^{*}=\inf b^{*}(\sigma)>0$. Moreover, by construction we have that $\Delta \mathrm{h}(\sigma, b)>0$ for any $b>b^{*}$. Associated to such condition one can find a critical leakage $\varepsilon^{*}$ such that

$$
\begin{array}{lll}
\text { if } & \varepsilon>\varepsilon^{*} & \text { horn-like region } \\
\text { if } & \varepsilon \leq \varepsilon^{*} & \text { strip-like region }
\end{array}
$$


where $\varepsilon^{*}=\sup _{\sigma}(\varepsilon(\sigma))$, where $\varepsilon(\sigma)$ is of the form:

$$
\varepsilon(\sigma)=1-\frac{\bar{x}_{2}-\bar{x}_{1}}{\rho\left(\bar{x}_{1}\right) \bar{x}_{2}-\rho\left(\bar{x}_{2}\right) \bar{x}_{1}}
$$

Note that $b^{*}=0$ for $\varepsilon(\sigma)$. Next we show that the expression (B5) is an increasing function of $\sigma$ and use this result to compute $\varepsilon^{*}$.

Proposition B.1 $\varepsilon(\sigma)$ is a strictly increasing function.

Proof: Let $y$ be the linear function given by

$$
y(x):=\rho\left(\bar{x}_{1}\right)+\frac{\rho\left(\bar{x}_{2}\right)-\rho\left(\bar{x}_{1}\right)}{\bar{x}_{2}-\bar{x}_{1}}\left(x-\bar{x}_{1}\right),
$$

where $\bar{x}_{i}=\varphi_{i}(\sigma)$. Note that the graph of $y$ is a straight line that passes through the points $\left(\bar{x}_{i}, \rho\left(\bar{x}_{i}\right), i=1,2\right.$, and that

$$
y(0)=\frac{\rho\left(\bar{x}_{1}\right) \bar{x}_{2}-\rho\left(\bar{x}_{2}\right) \bar{x}_{1}}{\bar{x}_{2}-\bar{x}_{1}}>1
$$

Moreover, we have

$$
\varepsilon(\sigma)=1-\frac{1}{y(0)}
$$

where $y(0)$ depends of sigma $\left(\bar{x}_{i}\right.$ depends of sigma). Proving that $y(0)$ is a strictly increasing function of $\sigma$, we have that $\varepsilon(\sigma)$ is also strictly increasing.

Next, by applying the mean value theorem (MVT) to $\rho(x)$, there exists $c \in\left(\bar{x}_{1}, \bar{x}_{2}\right)$ such that

$$
\frac{\rho\left(\bar{x}_{2}\right)-\rho\left(\bar{x}_{1}\right)}{\bar{x}_{2}-\bar{x}_{1}}=\rho^{\prime}(c) .
$$

Moreover, since $\rho$ is concave in $\left(\bar{x}_{1}, x^{*}\right)$ and convex in $\left(x^{*}, \bar{x}_{2}\right)$ there exist $x_{y} \in\left(\bar{x}_{1}, \bar{x}_{2}\right)$ such that $\rho\left(x_{y}\right)=y\left(x_{y}\right)$. So we can apply the MVT twice and get two points $c_{1} \in\left(\bar{x}_{1}, x_{y}\right)$ and $c_{2} \in\left(x_{y} \bar{x}_{2}\right)$ satisfying (B9). Moreover, $\rho^{\prime}\left(c_{1}\right)=\rho^{\prime}\left(c_{2}\right)$ and $c_{1} \in\left(\bar{x}_{1}, \min \left(x_{y}, x^{*}\right)\right)$ and $c_{2} \in\left(\max \left(x_{y}, x^{*}\right), \bar{x}_{2}\right)$. Indeed, as $\varphi_{1}$ is an strictly increasing function of $\sigma$ then there exists $\bar{\sigma}>\sigma$ such that $c_{1}=\varphi_{1}(\bar{\sigma})$.

Next, note that the constant slope of the graph of $y$ can be parameterized in terms of $\sigma$ and is given by

$$
m(\sigma)=\frac{\rho\left(\varphi_{2}(\sigma)\right)-\rho\left(\varphi_{1}(\sigma)\right.}{\varphi_{2}(\sigma)-\varphi_{1}(\sigma)}
$$

so that 


$$
\frac{d m}{d \sigma}(\sigma)=\frac{\left[\rho^{\prime}\left(\varphi_{2}\right) \varphi_{2}^{\prime}-\rho^{\prime}\left(\varphi_{1}\right) \varphi_{1}^{\prime}\right]\left(\varphi_{2}-\varphi_{1}\right)-\left(\rho\left(\varphi_{2}\right)-\rho\left(\varphi_{1}\right)\right)\left(\varphi_{2}^{\prime}-\varphi_{1}^{\prime}\right)}{\left(\varphi_{2}-\varphi_{1}\right)^{2}}(\sigma)
$$

Since $\rho^{\prime}\left(\varphi_{2}\right)=\rho^{\prime}\left(\varphi_{1}\right)$, by reordering terms we get

$$
\frac{d m}{d \sigma}(\sigma)=\frac{\varphi_{2}^{\prime}-\varphi_{1}^{\prime}}{\varphi_{2}-\varphi_{1}}\left[\rho^{\prime}\left(\varphi_{1}\right)-\frac{\rho\left(\varphi_{2}\right)-\rho\left(\varphi_{1}\right)}{\varphi_{2}-\varphi_{1}}\right](\sigma) .
$$

As $\varphi_{2}$ is decreasing and $\varphi_{1}$ is increasing, the first factor in Eqn (B11) is negative. Next, we prove that the second factor is positive in order to guarantee that $m$ is a decreasing function of $\sigma$. For this purpose, we consider that, $\varphi_{1}(\bar{\sigma})>\varphi_{1}(\sigma)$ and (as $\rho^{\prime}(x)$ is decreasing in $\left.\left(0, x^{*}\right)\right)$ we have:

$$
\rho^{\prime}\left(\varphi_{1}(\sigma)\right)>\rho^{\prime}\left(\varphi_{1}(\bar{\sigma})\right)=\frac{\rho\left(\varphi_{2}(\sigma)\right)-\rho\left(\varphi_{1}(\sigma)\right)}{\varphi_{2}(\sigma)-\varphi_{1}(\sigma)}
$$

Therefore, $m$ is a strictly decreasing function, thus $y(0)$ is a strictly increasing function of $\sigma$, thus ending the proof that $\varepsilon(\sigma)$ is a strictly increasing function of $\sigma$.

We have defined $\varepsilon^{*}=\sup (\varepsilon(\sigma))$, but $\varepsilon(\sigma)$ is a strictly increasing function then $\varepsilon^{*}=$ $\lim _{\sigma \rightarrow 1}(\varepsilon(\sigma))$.

\section{Proposition B.2}

$$
\varepsilon^{*}=\lim _{\sigma \rightarrow 1}(\varepsilon(\sigma))=\left(\frac{H+1}{H-1}\right)^{2}
$$

Proof: We have defined $\bar{x}_{1}:=\varphi_{1}$ and $\bar{x}_{2}:=\varphi_{2}$, where $\varphi_{1}(\sigma) \in\left(\begin{array}{ll}0 & x^{*}\end{array}\right)$ is a strictly increasing function of $\sigma$ and $\varphi_{2}(\sigma) \in\left(x^{*} \infty\right)$ is a strictly decreasing function of $\sigma$. Moreover we know

$$
x^{*}=K \sqrt[H]{\frac{H-1}{H+1}}
$$

then

$$
\rho\left(\bar{x}_{i}\right)=\frac{\bar{x}_{i}^{H}}{\bar{x}_{i}^{H}+K^{H}}=\frac{\varphi_{i}^{H}}{\varphi_{i}^{H}+K^{H}}
$$

Replacing in $\varepsilon(\sigma)$ the values of $\bar{x}_{i}$ we obtain

$$
\frac{\bar{x}_{1}-\bar{x}_{2}}{\rho\left(\bar{x}_{1}\right) \bar{x}_{2}-\rho\left(\bar{x}_{2}\right) \bar{x}_{1}}=\frac{\varphi_{1}-\varphi_{2}}{\rho\left(\bar{x}_{1}\right) \varphi_{2}-\rho\left(\bar{x}_{2}\right) \varphi_{1}}
$$

Replacing in the last expression the value of $\rho\left(\bar{x}_{i}\right)$ and arranging terms 


$$
\frac{\bar{x}_{1}-\bar{x}_{2}}{\rho\left(\bar{x}_{1}\right) \bar{x}_{2}-\rho\left(\bar{x}_{2}\right) \bar{x}_{1}}=\frac{\left(\varphi_{1}-\varphi_{2}\right)\left(\varphi_{1}^{H}+K^{H}\right)\left(\varphi_{2}^{H}+K^{H}\right)}{\varphi_{1}^{H} \varphi_{2}^{H}\left(\varphi_{2}-\varphi_{1}\right)+K^{H}\left(\varphi_{2} \varphi_{1}^{H}-\varphi_{1} \varphi_{2}^{H}\right)}
$$

The next equality is verified

$$
\varphi_{2} \varphi_{1}^{H}-\varphi_{1} \varphi_{2}^{H}=\varphi_{1}^{H} \varphi_{2}^{H}\left(\varphi_{2}^{-H+1}-\varphi_{1}^{-H+1}\right)=-\varphi_{1}^{H} \varphi_{2}^{H}\left(\varphi_{1}-\varphi_{2}\right) q\left(\varphi_{1}, \varphi_{2},-H\right)
$$

where

$$
q\left(\varphi_{1}, \varphi_{2},-H\right)=\varphi_{1}^{-H}+\varphi_{1}^{-H-1} \varphi_{2}+\cdots+\varphi_{1} \varphi_{2}^{-H-1}+\varphi_{2}^{-H}
$$

Then we can eliminate the term $\left(\varphi_{1}-\varphi_{2}\right)$ to obtain

$$
\frac{\bar{x}_{1}-\bar{x}_{2}}{\rho\left(\bar{x}_{1}\right) \bar{x}_{2}-\rho\left(\bar{x}_{2}\right) \bar{x}_{1}}=\frac{\left(\varphi_{1}^{H}+K^{H}\right)\left(\varphi_{2}^{H}+K^{H}\right)}{-\varphi_{1}^{H} \varphi_{2}^{H}-\varphi_{1}^{H} \varphi_{2}^{H} q\left(\varphi_{1}, \varphi_{2},-H\right) K^{H}}
$$

When $\sigma \rightarrow 1, \varphi_{1} \rightarrow x^{*}, \varphi_{2} \rightarrow x^{*}$ and $q\left(\varphi_{1}, \varphi_{2},-H\right)=(-H+1)\left(x^{*}\right)^{-H}$ then

$$
\begin{aligned}
\lim _{\sigma \rightarrow 1}(\varepsilon(\sigma)) & =1+\frac{\left(\left(x^{*}\right)^{H}+K^{H}\right)^{2}}{-\left(x^{*}\right)^{2 H}-\left(x^{*}\right)^{H} K^{H}(-H+1)}=1+\frac{K^{2 H}\left(\frac{H-1}{H+1}+1\right)^{2}}{K^{2 H}\left[\frac{H-1}{H+1}(H-1)-\left(\frac{H-1}{H+1}\right)^{2}\right]} \\
& =1+\frac{4 H^{2}}{H(H-1)^{2}}=\frac{(H-1)^{2}+4 H}{(H-1)^{2}}=\frac{H^{2}-2 H+1+4 H}{(H-1)^{2}}=\frac{(H+1)^{2}}{(H-1)^{2}}
\end{aligned}
$$

and it follows that $\varepsilon^{*}=\frac{(H+1)^{2}}{(H-1)^{2}}$.

[1] D. T. Gillespie, Annu. Rev. Phys. Chem. 58, 35 (2007).

[2] T. B. Kepler and T. C. Elston, Biophys. J. 81, 3116 (2001).

[3] H. McAdams and A. Arkin, Proc. Natl. Acad. Sci. U.S.A. 94, 814 (1997).

[4] M. B. Elowitz, A. J. Levine, E. D. Siggia, and P. S. Swain, Science 297, 1183 (2002).

[5] E. M. Ozbudak, M. Thattai, I. Kurtser, A. D. Grossman, and A. van Oudenaarden, Nature Genet. 31, 69 (2002).

[6] W. J. Blake, M. Kærn, C. R. Cantor, and J. J. Collins, Nature 422, 633 (2003).

[7] M. Kærn, T. C. Elston, W. J. Blake, and J. J. Collins, Nat. Rev. Genet. 6, 451 (2005).

[8] L. Cai, N. Friedman, and X. S. Xie, Nature 440, 358 (2006).

[9] X. S. Xie, P. J. Choi, G. Li, K. L. Nam, and G. Lia, Annu. Rev. Biophys. 37, 417 (2008).

[10] D. R. Larson, D. Zenklusen, B. Wu, J. A. Chao, and R. H. Singer, Science 332, 475 (2011). 
[11] Z. S. Singer, J. Yong, J. Tischler, J. A. Hackett, A. Altinok, M. A. Surani, L. Cai, and M. B. Elowitz, Mol. Cell 55, 319 (2014).

[12] M. Thattai and A. Van Oudenaarden, Proc. Natl. Acad. Sci. U.S.A. 98, 8614 (2001).

[13] J. Paulsson and M. Ehrenberg, Phys. Rev. Lett. 84, 5447 (2000).

[14] J. Paulsson, Nature 427, 415 (2004).

[15] N. Rosenfeld, M. B. Elowitz, and U. Alon, J. Mol. Biol. 323, 785 (2002).

[16] U. Alon, An Introduction to Systems Biology. Design Principles of Biological Circuits (Chapman \& Hall/ CRC, London, 2007).

[17] J. Paulsson, Phys. Life Rev. 2, 157 (2005).

[18] F. López-Caamal and T. T. Márquez-Lago, PLoS One 9, e103521 (2014).

[19] B. Munsky and M. Khammash, J. Chem. Phys. 124, 1 (2006).

[20] M. S. Sherman and B. A. Cohen, PLoS Comput. Biol. 10, e1003596 (2014).

[21] R. Yvinec, C. Zhuge, J. Lei, and M. C. Mackey, J. Math. Biol. 68, 1051 (2014).

[22] C. Jia, M. Qian, Y. Kang, and D. Jiang, Quant. Biol. 2, 110 (2014).

[23] C. Zechner, J. Ruess, P. Krenn, S. Pelet, M. Peter, J. Lygeros, and H. Koeppl, Proc. Natl. Acad. Sci. U.S.A. 109, 8340 (2012).

[24] G. Neuert, B. Munsky, R. Z. Tan, L. Teytelman, M. Khammash, and A. van Oudenaarden, Science 339, 584 (2013).

[25] M. Acar, J. T. Mettetal, and A. van Oudenaarden, Nature Genet 40, 471 (2008).

[26] M. Leisner, K. Stingl, E. Frey, and B. Maier, Curr. Opin. Microbiol. 2008, 553 (2008).

[27] M. C. Mackey, M. Tyran-Kamiska, and R. Yvinec, J. Theor. Biol 274, 84 (2011).

[28] A. Y. Mitrophanov and E. A. Groisman, Bioessays 30, 542 (2008).

[29] N. Friedman, L. Cai, and X. S. Xie, Phys. Rev. Lett. 97, 168302 (2006).

[30] V. Shahrezaei and P. S. Swain, Proc. Natl. Acad. Sci. U.S.A. 105, 17256 (2008).

[31] A. Ochab-Marcinek and M. Tabaka, Phys. Rev. E 91, 012704 (2015).

[32] R. D. Dar, B. S. Razooky, A. Singh, T. V. Trimeloni, J. M. McCollum, C. D. Cox, M. L. Simpson, and L. S. Weinberger, Proc. Natl. Acad. Sci. U.S.A. 109, 17454 (2012).

[33] L. Huang, Z. Yuan, P. Liu, and T. Zhou, BMC Syst. Biol. 9, 16 (2015).

[34] M. B. Elowitz and S. Leibier, Nature 403, 335 (2000).

[35] D. M. Suter, N. Molina, D. Gatfield, K. Schneider, U. Schibler, and F. Naef, Science 332, $472(2011)$. 
[36] A. Raj, C. S. Peskin, D. Tranchina, D. Y. Vargas, and S. Tyagi, PLoS. Biol. 4, 1707 (2006).

[37] D. T. Gillespie, J. Comput. Phys. 22, 403 (1976).

[38] V. Elgart, T. Jia, A. T. Fenley, and R. Kulkarni, Phys. Biol. 8, 046001 (2011).

[39] S. Hassani, Mathematical Methods for Students of Physics and Related Fields, 2nd ed. (Springer, New York USA, 2009). 